\title{
Lawful Humean explanations are not circular
}

\author{
Callum Duguid ${ }^{1}$
}

Received: 17 April 2020 / Accepted: 22 January 2021 / Published online: 6 February 2021

(c) The Author(s) 2021

\begin{abstract}
A long-standing charge of circularity against regularity accounts of laws has recently seen a surge of renewed interest. The difficulty is that we appeal to laws to explain their worldly instances, but if these laws are descriptions of regularities in the instances then they are explained by those very instances. By the transitivity of explanation, we reach an absurd conclusion: instances of the laws explain themselves. While drawing a distinction between metaphysical and scientific explanations merely modifies the challenge rather than resolving it, I argue that it does point us towards an attractive solution. According to Humeanism, the most prominent form of the regularity view, laws capture information about important patterns in the phenomena. By invoking laws in scientific explanations, Humeans are showing how a given explanandum is subsumed into a more general pattern. Doing so both undermines a principle of transitivity that plays a crucial role in the circularity argument and draws out a central feature of the Humean approach to scientific explanation.
\end{abstract}

Keywords Laws of nature · Grounding · Explanation · Humeanism · Lewis

\section{Introduction}

One of the most influential accounts of laws of nature is the regularity account, which takes the laws to be descriptions of regularities that occur in the world. This is most commonly associated with Humeanism, understood here as a rejection of irreducible necessity in nature. However, the Humean approach to laws has long been subject to the criticism that the laws it offers fail to back the sorts of explanation that we standardly take laws to be involved in. ${ }^{1}$ This criticism has recently seen a resurgence of interest, and a corresponding flurry of papers have been published on

\footnotetext{
1 A classic early statement of this is Armstrong (1983, p. 102).

Callum Duguid

calduguid@gmail.com

1 School of Philosophy, Religion and History of Science, University of Leeds, Leeds LS2 9JT, UK
} 
the matter. ${ }^{2}$ The aim of this paper is to identify a crucial feature of Humean laws that underwrites their ability to support the explanations we find in both folk and scientific practice. Since the resulting explanations do not support a principle of transitivity crucial to the circularity objection, I claim that this feature is one which all successful Humean responses to the challenge ought to share in. By focusing on this commonality in Humean accounts rather than the specifics of one in particular, it is hoped that this defence will be compatible with a range of pre-existing commitments. ${ }^{3}$

While this is an area of recent interest, the debate is a long-standing one and so suffers from differences in how its participants set up the problem, make distinctions and express their own claims. As such, the early sections of the paper will be primarily focused on laying out exactly what the main issue at stake is. Only once this has been accomplished will we be in a position to show that Humeans can use the notion of pattern subsumption to respond to the circularity argument. The next section begins with a recap of the most well-known Humean account of laws, the Best System Account, and then turns to the motivation behind the accusations of circularity. Barry Loewer's distinction between metaphysical and scientific explanations has shaped much of the recent debate in this area and Sect. 3 offers an illustration of this important distinction. In response to Loewer, it has been argued that these forms of explanation are connected by a principle of transitivity. Since we can chain metaphysical and scientific explanations together to form new scientific explanations, the Humean account is still subject to a revised circularity argument (Sect. 4). The advantage to approaching the issue in this way is that it clearly indicates what the challenge is: Humeans must reject one of the premises of the revised circularity argument without thereby having to reject all cases of chained explanations that occur in scientific practice.

The paper then shifts to a diagnosis of the central issue and argues that the sense of explanation at play is an inappropriately strong one (Sect. 5). In particular, it requires the laws appealed to by an explanation to play a non-Humean role: they must be responsible for the mosaic's being the way that it is. As there is another sense of explanation available, one based on identifying events as instances of a broader pattern, Humeans are free to reject the one present in the circularity argument. Moreover, given the Humean conception of what roles laws are capable of playing and which aspects of the world are explicable, they have motivation to do so that is independent of the circularity debate. In Sect. 6, the paper then connects this proposal to three more specific accounts of scientific explanation and demonstrates that each can make sense of the chaining examples used to motivate the transitivity principle. The conclusion reiterates the central claim of this proposal: Humean

\footnotetext{
${ }^{2}$ Lange (2013) provides the main contemporary case against Humean laws and explanations. Hicks and van Elswyk (2015), Marshall (2015) and Miller (2015) offer responses. Lange (2018) replies to these defences.

3 And so might be contrasted with more controversial solutions, like the 'contrarian' Humean in Miller (2015).
} 
explanations have pattern subsumption as a central characteristic and this feature undermines the transitivity principle that the circularity argument depends upon. ${ }^{4}$

\section{Humeanism and the circularity argument}

The Humean denial of necessary connections blocks off certain views about the nature of scientific laws. As metaphysically productive concepts like governance are not obviously available on this view, Humeanism is associated with regularity accounts of law. ${ }^{5}$ The most popular way of then distinguishing between lawful and accidental regularities is the Best System Account. ${ }^{6}$ According to this account, the various ways in which we can systematise truths about the world differ with respect to how informative and how simple they are. These two virtues trade off against each other, as including more facts about the world strengthens the system at the price of making it less simple. The system that achieves the best balance of theoretical virtues gives us the laws. More specifically, the laws are the universal generalisations included as axioms in the best systematisation of the world's facts. Let's call the collection of facts that determine the laws 'the mosaic'?

One of the roles commonly ascribed to laws is that they must be able to explain their instances. But, goes the objection, this is something that the regularity view of laws cannot give us. The core worry is that the laws are what they are in virtue of the way the world is, and hence they cannot explain why the world is that way. Attempts to do so are guilty of circular reasoning. If the fundamental state of the world explains the fundamental laws, then we cannot immediately turn around and explain the world's state by appeal to these very laws. Otherwise we would be claiming that the fundamental state of the world explains itself, only with the additional use of laws as an intermediary.

This is an objection commonly voiced by anti-Humeans. ${ }^{8}$ It has some intuitive force to it. Take Coulomb's law, which describes the electrostatic force between charged particles. A straightforward consequence of the law is that all positively

\footnotetext{
4 The phrasing of pattern subsumption might suggest a unificationist reading of this proposal but the terminology is more widely applicable. Hempel (1965, p. 488) describes the deductive-nomological account in terms of subsumption under a system of uniformities. Woodward (2003, p. 191) summarises explanation on the counterfactual account as "exhibiting systematic patterns of counterfactual dependence'. As neither of these views are standardly understood as unificationist, I take pattern subsumption to be a broader 'umbrella' term that covers views which see unification as the aim of explanation in addition to views which see it as aiming at other ends.

5 See Beebee (2000). For an argument that it is still possible to maintain that Humean laws govern in an interesting sense, see Roberts (2008).

6 See Lewis (1983, pp. 365-368). While the Best System Account has been criticised and refined in various ways, the basic form is sufficient for the purposes of this paper.

7 The aim of this broad characterisation is to avoid the controversies about the precise nature of the facts within the mosaic. While the connection between the mosaic and the laws was originally stated in terms of supervenience, it is not uncommon to see contemporary Humeans talk in terms of grounding. See, for example, Bhogal and Perry (2017) and Loew and Jaag (2020).

8 For example, Lange (2013, p. 256) and Maudlin (2007, p. 172).
} 
charged particles repel one another. Suppose that, upon considering some experimental setup, I were to ask why the two particles in the experiment repelled one another. Our usual way of thinking about scientific laws suggests an immediate answer to the question: these particles repel each other because both are positively charged and it is a law that positively charged particles repel. ${ }^{9}$ In other words, an instance of the law is explained by appeal to that law. However, anyone who provides an account of laws that takes them to be regularities faces a problem here. If the law that all positively charged particles repel each other is a law because it is a regularity-perhaps one that possesses other features, such as being a part of the best systematisation of the world's facts-then the reason it holds at all is ultimately because of the fact that there are positively charged particles and the fact that they repel each other. But if the reason that these particles repel is because of the law and the reason that there is such a law is because of the repelling particles, then we have an explanatory circle going on. For one of the law's instances is a partial explanation of the law and so at least a partial explanation of itself. And self-explanation is a high cost for Humeanism to bear, perhaps one that indicates that we do not have a genuine explanation of the phenomenon after all.

In order to assess the options open to Humeans, it will be useful to have a clear statement of exactly what this argument for explanatory circularity is. Hicks and van Elswyk provide a particularly clear formalisation of the objection:

(P1) The natural laws are generalisations.

(P2) The truth of generalisations is (partially) explained by their positive instances.

(P3) The natural laws explain their instances.

(P4) If $A$ (partially) explains $B$ and $B$ (partially) explains $C$, then $A$ (partially) explains $C$.

(C1) The natural laws are (partially) explained by their positive instances.

(C2) The instances of laws explain themselves (Hicks and van Elswyk 2015, p. 434).

While it might be more accurate to say that the instances of laws (partially) explain themselves, the problem this argument raises for the Humean account is evident. Even partial self-explanation would be an undesirable consequence of a regularity theory of laws. Worse, the tension between the Humean grounding of laws in the mosaic and transitivity allows problem cases to multiply. If the reason why these particles repel is because it is a law that all positively charged particles do so, and this is a law partially because of each instance of positively charged particles moving in such a manner, then the fact that particles a century from now will repel one another is part of the explanation why these particles do so. ${ }^{10}$ This is a strong claim,

\footnotetext{
${ }^{9}$ One might supplement these two claims according to one's favoured account of explanation. For example, on Woodward's (2003) counterfactual account of explanation, one would also point out that the lawfulness of this generalisation is relevant because it tells us a counterfactual dependence relation has been captured. As such, Coulomb's law allows us to answer a range of what-if-things-had-been-different questions.

10 This is suggested by Miller (2015, pp. 1324-1325) and echoed by both Marshall (2015, p. 3152) and Lange (2018).
} 
and at the very least it would be better for Humeans to not be forced into accepting it.

\section{Metaphysical and scientific explanations}

According to one influential response to this argument, for Humeanism about laws to be guilty of an explanatory circle, the explanations appealed to must be of the same kind. If we use $A$ to explain $B$ and then use $B$ to explain $A$ in the same kind of way, then, intuitively, there is something wrong with the explanation being offered. But if $B$ is used to explain $A$ in a different kind of way from the first case, then intuition may not tell against this. To put it another way, we might be prepared to grant that the transitivity principle in (P4) of the formalised argument holds for one kind of explanation, but resist the claim that transitivity will hold across different kinds of explanation. That is exactly the approach that I will describe in this section.

For this to work when applied to laws, of course, we will need to see some details as to exactly what the different kinds of explanation appealed to are supposed to be. Loewer (2012, p. 131) has suggested that there is such a distinction between metaphysical and scientific explanations. ${ }^{11}$ Metaphysical explanations are concerned with constitution in some way. To say that $A$ metaphysically explains $B$ is to say that $B$ is grounded in $A$, or that $B$ holds in virtue of $A$ or that $B$ is constituted by $A .{ }^{12}$ An explanation of this sort does not need to cite laws. If explanans and explanandum are both temporal entities, then they must be co-temporal. This latter point captures the following idea: if we want to say that $A$ is the underlying stuff out of which we get $B$ then they clearly must both exist at the same time. It would make little sense to claim that $B$ is constituted by something no longer around.

Scientific explanations do not need to mention grounds in that way. Rather, Loewer suggests that the core of scientific explanation of some event is showing why that event occurred through appeal to other events and certain laws. If $B$ scientifically explains $A$, then events mentioned in $B$ will typically be temporally prior to events in $A$. That said, this is not a strict requirement since there is no requirement that the explanans or the explanandum be temporally located entities. Another difference between the two kinds of explanation is that scientific explanations may be probabilistic while metaphysical ones cannot. The thermodynamic explanation of the melting of an ice cube in warm water proceeds by way of showing that this melting is highly probable, yet there does not seem to be an equivalent for constitutive explanations: if we claim that the water in that ice cube consists of $\mathrm{H}_{2} \mathrm{O}$ molecules, notions like chance or probability do not appear to have any role to play.

This is far from a full account of scientific explanation and is likely inspired by features drawn from different accounts. That said, it is not intended to be a single complete account. Loewer is instead trying to give some characterisation of what

\footnotetext{
11 See also his (2007).

12 More details about the contemporary notion of grounding can be found in Fine (2012) and Rosen (2010). The link between grounding and explanation specifically is considered in Jansson (2016).
} 
the kind of scientific explanation he has in mind is. To see how it is distinct from metaphysical explanation, let's consider the example of Arbuthnot's regularity. ${ }^{13}$ The regularity which forms our explanandum is simple: over an 82-year span from 1623 onwards, more boys than girls were born in London. Calling this mere coincidence is hardly credible given how unlikely this is to have come about by chance. But there are two kinds of explanation on offer for this regularity. Take the metaphysical explanation first. We may not have such an explanation presently available, but we can sketch out the form it might take. Begin by taking the exact states of all of the fundamental physical particles across 1623. Associate some distributions of particles with the birth of a boy and some other distributions with the birth of a girl. Count the number of boy-birth distributions and compare it to the number of girlbirth distributions. Repeat for the remaining 81 years. We can clearly see that more boys than girls were born in London each year!

There is a sense in which this constitutes an explanation of the Arbuthnot regularity. We have given the details (or, at least, the form such details take) of what the regularity might fundamentally consist in. The regularity obtains in virtue of suchand-such fundamental physical facts. But it is also easy to see what this explanation misses. Most obviously, it is an 'in principle' explanation: we do not have epistemic access to the world's fundamental state for any of the relevant years nor we do not believe we have an account of what a childbirth distribution would fundamentally consist in. However, even if we had all of this information available to us, the explanation would still be missing out on something. The offered explanation does nothing to help us understand why the regularity is not some big coincidence: why this particular fundamental distribution that generates the Arbuthnot regularity when so many others are possible? Furthermore, the explanation explains too much. For this appeal to a fundamental distribution not only explains the Arbuthnot regularity but also every other event and regularity that occurs during the relevant time period. So the very same explanans will feature in an explanation of the Great Fire of London, the publication of Descartes' Meditations, and the rise and fall of alien empires in other galaxies. The sense in which this is an explanation of the Arbuthnot regularity is perhaps a rather slim one.

So, there is plenty of room for an alternative to the metaphysical explanation just given. Contrast this with Fisher's neo-Darwinian explanation of the regularity. In any population which differs from a 1:1 ratio at sexual maturity, there is a selection pressure in favour of producing more offspring of the sex that is not as well represented. If one sex in a population is more vulnerable than the other, and so less likely to reach sexual maturity, natural selection will favour a birth ratio skewed towards the more vulnerable sex. In humans, males are more vulnerable than females: boys are more likely to die before puberty than girls. Any large human population which fails to birth more boys will experience a selection pressure towards having more

\footnotetext{
13 A discussion of Arbuthnot's regularity and explanation can be found in Kitcher (2001, p. 71). See Frisch (2011) for further discussion. The relevance of this example to Loewer's distinction was first brought to my attention in a talk by John Roberts.
} 
boys. This is why Arbuthnot observed this regularity; because of natural selection, every year is likely to be a year in which more boys are born than girls.

There are several differences in the character of the explanations just sketched. On Fisher's explanation, the regularity in the London births no longer appears to be a coincidence, but rather something we should expect to hold. This is because it has greater predictive power than the metaphysical one: we can use it to form beliefs about what would happen in relevantly similar counterfactual situations. Relatedly, it is less sensitive to the precise details of the background conditions. While neither explanation lends itself well to aiding us in manipulating the world so as to have brought about a different outcome, the scientific explanation does a better job of highlighting which features would need to be intervened upon if this were to occur. ${ }^{14}$ The variety of the explananda covered also differs. The scientific explanation does explain more than just a regularity in seventeenth-century London-it explains similar birth trends across different places and times-but it explains far less than the metaphysical explanation does. By appealing to the entire fundamental state, the latter explains every regularity that occurs in that 82-year period.

\section{The revised circularity argument}

A challenging line of response is given by Lange (2013, 2018). Grant that there is a distinction between scientific and metaphysical explanations. Grant also that the holding of some explanatory relation between $A$ and $B$, and another explanatory relation between $B$ and $A$ does not automatically entail that either explanation is viciously circular. Even so, Loewer's distinction will not save the Humean account. The problem is that these two kinds of explanation are connected to one another by a transitivity principle. Here is the original statement of it:

If E scientifically explains [or helps to scientifically explain] $\mathrm{F}$ and $\mathrm{D}$ grounds [or helps to ground] E, then D scientifically explains [or helps to scientifically explain] F (Lange 2013, p. 256).

The motivation for this update to our first transitivity principle is straightforward. If $D$ is the ground of $E$, then $E$ obtains because of $D$. If metaphysical explanation is concerned with constitution, then we can say that $E$ is constituted by $D$. Any role that $E$ plays, it plays because of the way it is and $D$ is what makes $E$ the way it is. So if $E$ is involved in the scientific explanation of some other fact then $D$ must also scientifically explain that other fact.

This allows us the run the argument against Humeanism again. The laws scientifically explain the mosaic, while the mosaic metaphysically explains the laws. By the transitivity principle, the mosaic must scientifically explain whatever the laws scientifically explain as it is only in virtue of the mosaic being a certain way that we have these laws to explain with. Hence the mosaic scientifically explains itself.

14 The importance of manipulation and intervention to explanation is stressed in Woodward (2003). 
Bhogal makes the case that even if the Humean finds examples where this sort of explanatory transitivity fails, we still need an account of why this is sometimes taken to be possible:

For example, we can chain together (i) a scientific explanation of the facts about the energy of the particles in this room from facts about those particles ten minutes ago, with (ii) a metaphysical explanation of the fact about the current temperature of the room in terms of facts about the energy of the particles in the room, to form (iii) a larger scientific explanation of the fact about the temperature in this room from the facts about the particles ten minutes ago (Bhogal 2017, p. 3). ${ }^{15}$

Clearly this sort of chaining together of explanations to form a scientific explanation from the grounds of some other explanatory fact is sometimes done. This is prima facie motivation to think that Lange's transitivity principle-or something very much like it - does hold. But the importance of this example is not merely to motivate that principle. Rather, it gives us a better understanding of the challenge that the Humean faces: the project is not only to show that the transitivity principle does not hold generally, but to do so in a way that does not condemn all instances of such explanatory transitivity. It must remain possible, after the Humean has offered a defence of the distinction between different types of explanations, for this chaining together to sometimes be a legitimate move. Taking a straightforward example, this rules out claiming that each explanandum has a unique scientific explanans. An argument for this would rule out the transitivity principle, since $E$ and the grounds of $E$ would not be able to both scientifically explain some $F$, but would leave it completely mysterious as to why we think explanations like the one in Bhogal's example are legitimate.

With this discussion in mind, we can now consider the explanatory circle argument that Humeans adopting Loewer's distinction must face:

$\left(\mathrm{P} 1^{\prime}\right)$ The natural laws are generalisations.

$\left(\mathrm{P} 2^{\prime}\right)$ The truth of generalisations is (partially) metaphysically explained by their positive instances.

$\left(\mathrm{P}^{\prime}\right)$ The natural laws scientifically explain their instances.

(P4') If $A$ (partially) metaphysically explains $B$ and $B$ (partially) scientifically explains $C$, then $A$ (partially) scientifically explains $C$.

(C1') The natural laws are (partially) metaphysically explained by their positive instances.

(C2') The instances of laws (partially) scientifically explain themselves.

Let us take this to be the objection that Humeans are challenged to overcome. The question now is how best to do that and the next two sections consider what I take to be the most promising line of response. In brief, the argument is that Humean

\footnotetext{
15 I take the challenge raised by these chained explanations to be the same as that raised by the combined explanations of Roski (2018).
} 
explanations function by way of subsuming events into more general patterns. This is at odds with the conception of explanation that anti-Humeans are working with. Fortunately for Humeanism, pattern-based explanations do not support the transitivity principle in ( $\left.\mathrm{P}^{\prime}\right)$. The final section makes this more explicit by connecting the approach to particular accounts of explanation and showing how they can respond to Bhogal's challenge.

\section{Explanation as pattern subsumption}

We now turn to how to respond to the circularity challenge. Attempts have been made to question the direction of the grounding relations in play here or to reconsider the motivation for accepting the transitivity principle (Miller 2015; Hicks and van Elswyk 2015). However, I shall suggest that the problem lies in the notion of explanation being appealed to. The sense of explanation at play is a strong one and it is possible for Humeans to reject it as inappropriate given their wider commitments. Doing so opens up two possible routes of response to the circularity argument: (1) claim that laws are not able to explain their instances, or (2) develop an alternative view of explanation that allows for laws to explain their instances without thereby supporting a principle of transitivity. In what follows, I will set out both responses but focus more closely on the latter, as this is not in tension with scientific practice.

Much of the literature responding to the explanatory circle argument has avoided giving a full account of what scientific explanation involves. There are good pragmatic reasons to do so: tying one's response to a particular account of explanation creates problems if that account brings with it unanswered objections, and, further, Humeans themselves adopt a range of different accounts. It is undesirable to only respond to the circularity worry on behalf of those who are committed to the same particular details about explanation as oneself. However, despite the advantages of neutrality here, there is something substantial that we can say about the strength of the explanation involved: it is in conflict with the spirit of the Humean conception of the world. More specifically, advocates of the circularity argument are using a notion of explanation that requires the laws to play a role that Humeans themselves do not take the laws to be capable of playing. Consider the following quotations:

If one is a Humean, then the Humean Mosaic itself appears to admit of no further explanation. Since it is the ontological bedrock in terms of which all other existent things are to be explicated, none of these further things can really account for the structure of the Mosaic itself (Maudlin 2007, p. 172, italics in original).

The Humean reduction seems to leave us without anything that has the requisite governance or guidance needed for lawfulness, that is, for control over the pattern of property instantiation as the world evolves. ${ }^{16}$

\footnotetext{
16 Paul (2013, p. 100), italics added for emphasis. Paul is reporting a wider concern with Humean laws, of which the issue with explanation is a specific instance.
} 
In short, if the Humean mosaic is responsible for making certain facts qualify as laws, then the facts about what the laws are cannot be responsible for features of the mosaic (Lange 2013, p. 256, italics added for emphasis).

I take accounting for, control and responsibility to be different ways of capturing the same basic concept here. For simplicity, I'll talk in terms of responsibility in what follows. In the third quotation, the first sort of responsibility is the sort of responsibility that accompanies the grounding relation. Even if one is suspicious about grounding and distinctively metaphysical explanations, there is a clear sense in which Humeans take laws to be (partially) constituted by events in the mosaic. However, the second mention of responsibility is rather odd. On the Humean view, laws are mere descriptions. They do not govern or constrain or make anything happen. Of course they are not responsible for the mosaic; Humean laws are not responsible for anything! Ultimately, nothing is responsible for the mosaic being a certain way. It is a brute fact that the world is one with this pattern of events, as opposed to any other. This is captured by the famous Humean denial of necessary connections, the ban on which prevents anything from taking responsibility for the mosaic's turning out one way rather than another.

The second part of Lange's quote is picking out a core commitment of the Humean view, yet anti-Humeans take it to express an objection: that the laws are not responsible for the mosaic is supposed to be a problem. Perhaps the lack of responsibility that laws show is an unattractive feature of the account for its detractors. But that in itself is not a strong objection, especially when Humeans find the notion of responsible laws mysterious. There is a question here of who this account of scientific explanation is for. I take it that the account must be plausible to those who share in Humean presuppositions about the range of metaphysical resources available to work with. If an account must be attractive to even those who possess different worldviews, then we have set the bar too high for the vast majority of philosophical work to meet. A telling objection is one that its target will recognise as a challenge, whereas if the mosaic ultimately admits of no deeper explanation then the account's lack of one does not look like a deficiency.

A belligerent Humean might wish to push this point. The problem with the explanatory circle argument is that it assumes that laws can scientifically explain their instances in the sense of being responsible for them. Since explanation here is being meant in a strong sense, such a Humean does not take laws to be capable of doing that. In effect, this is a rejection of the argument's third premise. The explanatory circle is broken as one of the directions of explanation does not obtain. On this view there is no need to engage with the transitivity principle: while events in the mosaic metaphysically explain the laws, those laws do not scientifically explain the events and so there is no risk of the events scientifically explaining themselves.

Denying the third premise both blocks the circularity argument and fits in nicely with the Humean conception of laws as playing a descriptive role. But it comes at a great price, for this denial of their role in scientific explanations stands in conflict with both a commonsense belief about laws and how scientists are wont to use them. Perhaps the conflict with the folk usage can be explained away. After all, governance is commonly associated with laws but Humeans are committed to denying that 
they play that role. A degree of revisionism might be justified on the grounds that it comes with a more attractive metaphysics. But the ascription of error to working scientists is not so easily dealt with. One of the main motivations for adopting a Humean view is a respect for science and a suspicion of claims that science cannot grasp the nature of the world without some form of metaphysically weighty supplementation. ${ }^{17}$ To claim that scientists are misusing laws and should revise this usage on metaphysical grounds is, at the very least, in tension with this motivation. ${ }^{18}$

When confronted with the bullet that needs to be bitten on the route just described, many Humeans will wish instead for a more moderate line of response. Such a response will have to concede that laws can indeed help to explain their instances. It can, however, ask what reason there is to think that the kind of explanation appealed to in the argument is the kind of explanation that Humean laws can provide. In other words, if anti-Humeans like Lange are appealing to a 'thick' form of explanation in their objection, then it is open to Humeans to rescue the explanatory role of laws by appealing to a 'thinner' form of explanation. The question that then remains is what kind of explanation is involved in this thin form.

Recall that Humean laws are nothing more than particularly efficient ways of marking out important patterns in the world's events. Consequently, by Humean lights all scientific explanations that involve appeal to laws must thereby involve appeal to generalisations. To appeal to generalisations in this way is to draw the current explanandum into a wider network of events, treating it as another instance of a general pattern. In other words, scientific explanations that involve laws should be treated as cases of subsumption under a pattern.

Such acts of explanation are not unusual. If we relax our focus from explanations offered by working scientists, we can see that they are, in fact, commonplace. This raven is black because all ravens are black. I sit at the back of the lecture theatre because all of the cool kids sit at the back of the class. The All Blacks performed a haka before their last match because they always perform a haka before matches.

This provides a way to avoid being committed to laws being responsible for events without ascribing widespread error to scientists. The explanation Humeans can provide of the laws is a constitutive one: the laws are grounded in the patterns that occur in the mosaic. The explanation of the mosaic via the laws cannot be one in which the laws are taken to have any responsibility for the goings-on in the world. Rather, the explanations that invoke the laws are explanations in the form of pattern recognition. If I say that my pen falls because of gravity, I am pointing out that massive objects show a general pattern: they move towards one another. As my pen has mass, it is just another instance of this pattern. I have not attempted to identify something that acts on my pen to ensure that it moves downwards. Nor have I tried to find why this is a world with a gravity-pattern in it (that would presumably be an attempt to find a deeper reason that Lange takes appeals to regularities to be

\footnotetext{
17 This is particularly clear in Lewis (1994, p. 474).

18 See Emery (2019, pp. 1541-1544) for a defence of the claim that the laws explain their instances. The belligerent Humean described here has considerable work to do in offering a revisionary account of scientific practice.
} 
pointing towards). In fact, I had better not try to do this if I am a Humean! Ultimately, there is no deep reason why the world is one with this pattern of events as opposed to another one. For Humeans, there simply is no explanation of that kind of the mosaic to be had.

The importance of this line of reply is not merely that it utilises a different sense of explanation to that employed by anti-Humeans in the debate. That alone would be insufficient because the circularity argument might simply be reformed with reference to this thinner sense. Notions like responsibility are useful in describing the challenge but are not directly appealed to in the argument itself. Rather, the important point is that it undermines support for the argument's fourth premise: the transitivity principle. This principle has some plausibility when we think of explanations as offering something like causal information. If $E$ brings about $F$, and $E$ is really just made up from $D$, then it is not unreasonable to think that $D$ brings about $F$. That kind of neat story is not available when it is patterns that we are interested in. Suppose that $E$ explains $F$ by treating it as part of some wider pattern and that $E$ is grounded in $D$. There is nothing to guarantee that $D$ will be able to explain $F$ in the same way that $E$ does as patterns evident amongst the $E$-facts might not be apparent amongst the $D$-facts. Recall that for Humeans, the laws are determined by balancing descriptions of the entire mosaic via the Best System Account. When a scientific explanation is offered of some instance, that instance is being subsumed into the pattern captured by the law invoked in the explanation. If transitivity held, the instance would also be subsumed into a pattern at the level of the entire mosaic. But the whole mosaic is large and varied; it is not at all clear how it comprises a pattern under which any one instance could be subsumed. ${ }^{19}$

As this is clearer with an example, recall the contrasting explanations offered of Arbuthnot's birth-rate regularity. Here we have the lower-level explanation in terms of facts about the configuration of fundamental particles and the higher-level explanation in terms of a neo-Darwinian appeal to facts about vulnerability within a population and natural selection pressures. While both identify facts about the state of the world, we have already seen reason to prefer the latter one: the entire point of turning to an explanation like Fischer's is that the particle-based explanation does not properly capture what we're looking to get out of an explanation. The fact that the regularity still appears to be coincidental in an important sense in the lower-level explanation is evidence that the relevant pattern is not apparent when looking only at the lower-level facts.

This is still quite an abstract approach to the problem. The next section makes things more concrete by connecting this response to several positions on scientific explanation hospitable to Humean presuppositions. In doing so, the paper aims to show how these positions respond to the challenge raised by Bhogal and mentioned in the previous section. That is, to show how they can accommodate the kinds of examples used to motivate the transitivity principle without being required to accept it.

19 Thanks to Juha Saatsi for suggesting a clean way of expressing this point. 


\section{Humean views of explanation}

I have claimed both that the circularity argument against Humean laws requires a transitivity principle and that the Humean commitment to scientific explanations as pattern subsumption undermines such a principle. Yet Lange $(2013 ; 2018)$ has offered various examples to motivate precisely that principle. The fourth section of this paper mentioned an example from Bhogal (2017, p. 3):

(i) a scientific explanation of the facts about the energy of the particles in this room from facts about those particles ten minutes ago, with (ii) a metaphysical explanation of the fact about the current temperature of the room in terms of facts about the energy of the particles in the room, [forms] (iii) a larger scientific explanation of the fact about the temperature in this room from the facts about the particles ten minutes ago.

I took this to be a challenge and agreed that an account is owed of what is going on in examples like these. The aim of this section is to settle that account by showing that there are different ways of making sense of those explanations without thereby being committed to a troublesome transitivity principle.

Let's begin with the classic deductive-nomological account. Consider how Hempel (1965, p. 488) talks about explanation:

We noted that scientific explanation is not aimed at creating a sense of familiarity with the explanandum; "reduction to the familiar" is at best an incidental aspect of it. The understanding it conveys lies rather in the insight that the explanandum fits into, or can be subsumed under, a system of uniformities represented by empirical laws or theoretical principles...The central theme of this essay has been, briefly, that all scientific explanation involves, explicitly or by implication, a subsumption of its subject matter under general regularities; that it seeks to provide a systematic understanding of empirical phenomena by showing that they fit into a nomic nexus.

Note in particular the claim that scientific explanation involves subsumption under regularities. As has been discussed, this is a claim that Humeans should find themselves sympathetic to, given the Humean view of laws as particularly important regularities. Explanation on this account requires that the explanandum be derivable via a deductive argument from premises which essentially include at least one law. It's possible for such explanations to cross different domains, most obviously when we have a case of theoretical reduction like that of thermodynamics to statistical mechanics.

This is exactly what's going on in Bhogal's example, of course. A Humean who accepts the deductive-nomological account of explanation can grant that this is genuine case of scientific and metaphysical explanations being chained together. However, such a Humean need not grant that this gives us good reason to think that the transitivity principle holds. Rather, a more restricted principle holds: scientific explanation is transitive in this manner only when there exists a reduction of one theoretical domain to another. This is certainly not automatic-establishing the 
existence of a reduction takes considerable effort from practicing scientists. That's why the chaining of explanations is sometimes, but not always, possible. ${ }^{20}$ On this account of explanation, Humean explanations would only be circular if the relevant domain was reducible to the vocabulary of the mosaic, which solely describes perfectly natural properties connected by spacetime relations. Even though the mosaic is involved in the metaphysical explanation of all laws, particular instances of those laws are not scientifically explained by it since we do not have a principle that supports the chaining of those kinds of explanation in such situations.

In developing his unificationist account of explanation, Kitcher (1981, p. 508) quotes approvingly from Hempel (1966, p. 83):

What scientific explanation, especially theoretical explanation, aims at is not [an] intuitive and highly subjective kind of understanding, but an objective kind of insight that is achieved by a systematic unification, by exhibiting the phenomena as manifestations of common, underlying structures and processes that conform to specific, testable, basic principles.

This is taken to be the more promising unofficial view that underlies the deductivenomological account and forms the motivation for the unificationist one. This latter account claims that scientific explanation aims at unification, understood as the reduction of the number of facts that must be accepted as basic. From these basic facts, our explanations aim to derive as wide a range of kinds of events as possible. Put another way, the greater the range of phenomena that can be subsumed under a general pattern, the better the explanation. As has been noted by Woodward (2017), this fits nicely alongside a view that has laws occur in these explanations, and further claims that the laws are the result of a competition to find the best way to balance simplicity against comprehensiveness. If, for this reason, scientific explanation aims to unify by showing how a given explanandum is an instance of a law (and so demonstrates that the explanandum does not need to be accepted as basic), then it aims to draw that explanandum into a wider regularity. In doing so, the Humean unificationist is committed to basing scientific explanation around pattern subsumption. ${ }^{21}$

Perhaps unsurprisingly given its origins, the unificationist account judges Bhogal's example and the transitivity principle in a similar way to the

\footnotetext{
20 The reduction here cannot be merely an in-principle ontological reduction. As everything reduces to the fundamental mosaic, understanding reduction in that sense would do nothing to block the transitivity principle. What's required instead is a form of linguistic reduction that shows that the higher-level phenomenon is covered by the lower-level laws. Providing such a reduction demonstrates why there is a transitivity to the pattern subsumption here: as the higher-level regularities can be expressed in terms of the lower-level ones, phenomena deducible from the former regularities are also deducible from the latter regularities. Assuming the deduction meets the other requirements of the deductive-nomological account, we have a successful explanation at both levels. While controversial, Nagel's (1961) model of theoretical reduction both captures the relevant sense of reduction and aligns with the deductive-nomological account - further development in this area is an important desideratum for Humeans who adopt this view of explanation.

21 Armstrong (1983) takes unification to be a mark of explanation when criticising the regularity account of laws, although he does not acknowledge there the possibility of Humeans appealing to unificationist explanations.
} 
deductive-nomological account. A transitivity principle holds when the grounding facts have been shown to be more basic than the grounded ones, in the sense that the grounded phenomena have been unified under the grounding phenomena. In such cases we can derive the explanandum from the more basic facts. Again, showing that this is possible is not automatic and is not achieved from the comfort of the philosophy armchair. Chaining is possible in Bhogal's example because of the reduction of the thermodynamic facts. It is not possible in general since not all facts have been shown to be derivable from a set of basic ones. The more general principle required by the circularity argument itself requires the unification of all scientific domains under facts about the mosaic. This is something that we clearly have not achieved.

Both the unificationist and deductive-nomological accounts are well-established accounts of explanation compatible with a Humean approach to metaphysics. ${ }^{22}$ Given their broadly similar motivations however, one might worry that the approach advocated here is unnecessarily restrictive, leaving out other accounts of explanation that Humeans might adopt. While an analysis of every compatible account is beyond the scope of a single paper, I will now turn to a more recent proposal that differs in an important respect to those just covered.

Dorst (2019) suggests that to respond to the circularity argument we should reemphasise Loewer's distinction between metaphysical and scientific explanations. On the proposed view, the virtues invoked by the Best System Account that guide the choice of laws are taken to be constitutive of lawhood. In a similar vein, the explanatory virtues that guide theory choice are taken to be constitutive of theoretical truth: whichever theory best satisfies those virtues is true. We care about such virtues, and so about which theories are true, because of their predictive utility. So, in the scientific domain, theoretical truth drops out of predictive utility. In metaphysics, this is not the case. Without needing to take a stand on what the precise aims of metaphysics are, we can say that they are not based around predictive utility. As a result, the truth of metaphysical theories cannot be due to their supporting prediction.

Scientific and metaphysical explanations, then, are claimed to have different aims. This undercuts the motivation for thinking that the transitivity principle holds: why think that these two activities with different ends should always be linked in such a way? In the case of examples like Bhogal's, Dorst's view is that these are not mixtures of scientific and metaphysical explanations. Rather, these are scientific explanations being chained together to form another scientific explanation. ${ }^{23}$ The motivation for this claim is one we have already encountered: the reduction of

\footnotetext{
22 There are standard objections that have been raised to both; Woodward (2003) provides a thorough overview. In responding to the circularity argument by appealing to any favoured account of explanation, Humeans may be issuing a promissory note. It is the role of future research in this area to make good on that promise by further developing a plausible view of explanation that responds to the counterexamples.

23 Despite the view's motivations, this brings out an important difference between Dorst and Loewer in his (2012). The latter takes grounding to be a mark of metaphysical explanation, while the former allows grounding relations to occur in explanations that are solely scientific. Whether this price of accepting Dorst's view is a significant one depends on what one makes of the relationship between grounding and explanation more generally.
} 
thermodynamics to statistical mechanics is the result of scientific work rather than that of metaphysicians. In contrast, the grounding of the laws in the mosaic is clearly a metaphysical explanation: this claim forms no part of current scientific practice. As transitivity only applies across scientific explanations, the Humean grounding claim cannot be used to form a circle of scientific explanation.

A view of explanation like Dorst's does not explicitly appeal to patterns, but the same line of reasoning used in the previous section is at play here too. Even when $E$ scientifically explains $F$, the grounding of $E$ in $D$ does not ensure that $D$ thereby scientifically explains $F$. A further story is required, one taken from scientific practice that shows us how we can use the occurrence of $D$-facts in conjunction with laws to predict the occurrence of $F$-facts. As a Humean view, it is an underlying appeal to patterns that enables this prediction. We might not be able to predict an event because we know that relevant governing laws are responsible for bringing it about, but we can predict on the basis that it will be another instance of a wider pattern of similar events.

How similar is my proposal to a recent one by Bhogal (forthcoming) $?^{24}$ Like this paper, Bhogal also accepts Loewer's distinction between metaphysical and scientific explanation and finds reason to reject the transitivity premise in non-chaining cases. Similarly to Dorst, he does this by taking those explanations to be different activities with different aims. Metaphysical explanation is taken to be tied to grounding, while scientific explanation is understood as unificationism in the spirit of Friedman (1974). Since attempts to explain instances of laws by way of reference to the entire mosaic do not provide unification, they fail to count as genuine scientific explanations and so cannot serve as evidence of an explanatory circle.

As mentioned earlier in this section, I take my proposal to be compatible with the unificationist stance and so also with Bhogal's approach. However, there are some differences between our views. The most important is that this paper aims at neutrality between the various accounts of explanation on offer in the literature. While pattern subsumption fits well with a unificationist position on explanation, it is not tied to that account alone: the point of this section and the previous one has been to show why multiple extant accounts of explanation do not support a transitivity principle when combined with a Humean worldview. Transitivity might be supported by a form of scientific explanation associated with notions like responsibility, but the above accounts are examples of how we can approach explanation without such an association.

While Dorst and Bhogal make particular claims about the aims of science-prediction and unification respectively-this paper remains neutral. I claim that (lawful) scientific explanation has a pattern-subsuming feature in virtue of the descriptive nature of laws on the Humean account. However, this should not be taken as commitment to any one view regarding what science aims to achieve. The goal of this section has been to show that pattern-subsuming explanations can feature in accounts that take the activity of explaining to be aiming at deduction, unification or prediction. The reason for this form of neutrality is to aim for compatibility with

${ }^{24}$ I would like to thank an anonymous referee for pushing me on this connection. 
as wide a range of existing accounts of explanation as possible. ${ }^{25}$ A strength of this compatibility is that it shows how a variety of Humeans can respond to the circularity argument, as opposed to only those who have a shared conception of scientific goals. It means that Humeans have greater options regarding what to make of explanation and are not tied to the success or failure of a single account. ${ }^{26}$

\section{Conclusion}

While the threat of circularity has long dogged the Humean account of laws, this paper has argued that the objection is based on a view of explanation that Humeans are free to reject. Even stronger than that, Humeans have good reason to reject it as there is an alternative account that fits more closely with the commitment to laws as regularities. If the laws are descriptions of patterns, then scientific explanations invoking laws explain by way of showing that the explanandum in question is an element of some pattern. Explanations based on pattern subsumption do not support the circularity argument as they defy the transitivity principle: that our understanding of the explanandum is increased by seeing how it fits into a pattern amongst certain facts does not mean that there will be a similar increase in understanding from a description of the phenomenon in terms of other facts (and similarly for predictive utility).

In the fourth section, we saw that part of the challenge of accounting for how Humean laws explain was that we sometimes reason as if transitivity does hold. This was Bhogal's problem of chaining: if the transitivity principle does not hold generally, why is it that some metaphysical and scientific explanations can be chained together to form a legitimate scientific explanation? Specifically, why is it that we can chain together scientific facts about energy over time with metaphysical facts about the connection between energy and temperature to form a scientific explanation of a room's temperature over time?

The sixth section went some way to answer this, pointing out that exactly what is going on in the motivating examples depends on the view of explanation that one subscribes to. But in all cases, the grounding of some facts in certain other facts is not by itself sufficient to support the transitivity principle. Instead, something more is required, whether that be the unification of phenomena under a set of more basic

\footnotetext{
25 A further example of this is the counterfactual account of explanation, which has been rising in popularity (Woodward 2003; Saatsi and Pexton 2013; Reutlinger 2018). On this account, the purpose of scientific explanation is to enable us to answer what-if-things-had-been-different questions rather than to unify phenomena. The dependence relations underlying such answers are plausibly amenable to a Humean treatment but, as Bhogal is committed to unification being the aim of scientific explanation, he cannot avail himself of such a move. Neutrality has its advantages here.

${ }^{26}$ Bhogal in his (forthcoming) briefly suggests not being tied to 'surface level' unificationist accounts like those of Friedman or Kitcher. It might be that, like this paper, he intends something with wider appeal. Even if so, we still make different claims regarding the aim(s) of scientific explanation. This is why he treats Dorst's position as a competitor: it makes a different specific claim about what scientists are aiming at to Bhogal's. As this paper remains neutral on that issue, I am not forced into conflict with Dorst.
} 
facts, supporting the making of predictions, or something else entirely. The fact that some explanandum can be subsumed under a pattern does not imply that the grounds of that pattern will automatically serve as the scientific explanans of that explanandum.

This has not been an examination of scientific explanation in its full generality. The focus of the paper was rather on scientific explanations that make ineliminable appeal to laws. One might wonder whether there are any scientific explanations that do not involve laws and, if so, what a Humean might have to say about those. However, the circularity worry was not motivated by how Humeans treat scientific explanations in general, but by how they treat the laws that, at least some of the time, are involved in those explanations. In short, a scientific explanation that makes no mention of regularities will not be viciously circular since it lacks the allegedly circular feature. The question of how to treat such explanations remains, of course, but the answer is dependent upon one's views on scientific explanation more generally. The purpose of this paper is to argue that Humean theories of explanation should have a feature in common with each other, one that prevents them from being circular in the way anti-Humeans suggest. This is compatible with there being more than one theory of explanation available: individual Humeans should look to their favoured one to determine how they should treat these lawless cases.

In concentrating on the most influential recent formulation of the explanatory circle argument, I have not directly engaged with alternative formulations that might be raised. As an instructive example of how Humeans might adapt the position in this paper to those alternatives, I will comment briefly on Emery's (2019) argument. ${ }^{27}$ This is based on the conjunction of three claims:

(E1) Laws explain their instances.

(E2) Laws do not cause their instances.

(E3) If the $F$ s explain the $G$ s and the $F$ s don't cause the $G$ s, then the $F$ s ground the Gs.

From these it follows that the laws ground their instances. If successful, this argument would undermine Loewer's distinction as a way to respond to the circularity objection: as the laws are grounded in their instances and also ground their instances, Humeans are committed to circular grounding. Since grounding is tightly connected to metaphysical explanation, such a circle would result in metaphysical self-explanation. Setting aside the belligerent Humean of Sect. 5, Humeans have little quarrel with (E1) or (E2). The point of divergence is therefore the third claim. Emery does offer a defence of this, pointing out that it fits several examples of explanations in science and has an important virtue: it is the simplest plausible account on offer.

Plausibility matters here, since a simpler account is one which takes all explanation to be causation. As Emery (2019, p. 1546) rightly notes, the cost of accepting such an account is too high to be outweighed by the gain in simplicity. However, this opens the door for Humeans to adopt an account of scientific explanation from

27 Thanks to an anonymous referee for suggesting this comparison. 
the literature as indicated previously. Such accounts are independently motivated, forestalling accusations of this being an ad hoc move. They offer more sophisticated treatments of explanation than does the account present in (E3), offsetting the loss in simplicity by gains in how closely they reflect scientific practice or accommodate difficult examples. ${ }^{28}$ Perhaps Emery is right to think that there is some cost to accepting explanatory relations beyond causation and grounding. If so, it is one that many philosophers of science are willing to pay. Contemporary Humeans think that the mosaic serves as the grounding base for all the nonfundamental facts. While those facts are grounded in the mosaic, the mosaic itself is not grounded in any further facts-including those concerning laws. As such, Humeanism comes with a need to find an account of scientific explanation where laws do not ground their instances.

The Humean view is often associated with a suspicion of 'heavy-duty' metaphysics, the kind that introduces irreducible necessity into the world. The cost of avoiding such commitments is a thinness to the resulting explanations. To some, this cost will render the position unattractive: recall Lange's point about the laws not being responsible for features of the mosaic. The aim of this paper has not been to sway the anti-Humean camp on this. More modestly, it has been to show that if one is willing to accept Humean presuppositions about the world and the available metaphysical resources, then it is still possible to provide a sense in which laws can explain that does not require attributing widespread error to the scientific community. A consequence of the Humean account is that thicker explanations of the world's events are not available: it is ultimately a brute fact that we have this mosaic rather than any other. As such, the restriction to thinner explanations is no substantial cost to the Humean position. ${ }^{29}$

Acknowledgements I would like to thank Víctor Durà-Vilà, Steven French, Rob Knowles and Juha Saatsi for many helpful comments on earlier drafts of this paper.

Author contributions Single-authored.

\section{Compliance with ethical standards}

Conflict of interest The authors declare that they have no conflict of interest.

Open Access This article is licensed under a Creative Commons Attribution 4.0 International License, which permits use, sharing, adaptation, distribution and reproduction in any medium or format, as long as you give appropriate credit to the original author(s) and the source, provide a link to the Creative Commons licence, and indicate if changes were made. The images or other third party material in this article are included in the article's Creative Commons licence, unless indicated otherwise in a credit line to the material. If material is not included in the article's Creative Commons licence and your intended use is not permitted by statutory regulation or exceeds the permitted use, you will need to obtain permission

\footnotetext{
${ }^{28}$ Emery reminds us that various tricky cases are not what is going on when instances are being explained through appeal to laws. It is worth pointing out, however, that an account able to offer a unified treatment of scientific explanation has plausibly justified the loss of theoretical simplicity through its more parsimonious treatment of example cases.

29 Thanks are due to Víctor Durà-Vilà, Steven French, Rob Knowles, Juha Saatsi and several referees for their comments and discussions of this paper.
} 
directly from the copyright holder. To view a copy of this licence, visit http://creativecommons.org/licen ses/by/4.0/.

\section{References}

Armstrong, D. (1983). What is a law of nature? Cambridge: Cambridge University Press.

Beebee, H. (2000). The non-governing conception of laws of nature. Philosophy and Phenomenological Research, 61, 571-594.

Bhogal, H. (forthcoming). Nomothetic explanation and humeanism about laws of nature. Oxford Studies in Metaphysics.

Bhogal, H. (2017). Minimal anti-humeanism. Australasian Journal of Philosophy, 95, 447-460.

Bhogal, H., \& Perry, Z. (2017). What the Humean should say about entanglement. Nous, 51, 74-94.

Dorst, C. (2019). Humean laws, explanatory circularity, and the aim of scientific explanation. Philosophical Studies, 176, 2657-2679.

Emery, N. (2019). Laws and their instances. Philosophical Studies, 176, 1535-1561.

Fine, K. (2012). Guide to ground. In F. Correia \& B. Schneider (Eds.), Metaphysical grounding: Understanding the structure of reality (pp. 37-80). Cambridge: Cambridge University Press.

Friedman, M. (1974). Explanation and scientific understanding. The Journal of Philosophy, 71, 5-19.

Frisch, M. (2011). From Arbuthnot to Boltzmann: The past hypothesis, the best system, and the special sciences. Philosophy of Science, 78, 1001-1011.

Hempel, C. G. (1965). Aspects of scientific explanation. In C. G. Hempel (Ed.), Aspects of scientific explanation and other essays in the philosophy of science. New York: The Free Press.

Hempel, C. G. (1966). Philosophy of natural science. Englewood Cliffs, NJ: Prentice-Hall.

Hicks, M. T., \& van Elswyk, P. (2015). Humean laws and circular explanation. Philosophical Studies, 172, 433-443.

Jansson, L. (2016). Explanatory asymmetries, ground, and ontological dependence. Erkenntnis, 82, $17-44$.

Kitcher, P. (1981). Explanatory unification. Philosophy of Science, 48, 507-531.

Kitcher, P. (2001). Science, truth and democracy. Oxford: Oxford University Press.

Lange, M. (2013). Grounding, explanation, and Humean laws. Philosophical Studies, 164, 255-261.

Lange, M. (2018). Transitivity, self-explanation, and the explanatory circularity argument against Humean accounts of natural law. Synthese, 195, 1337-1353.

Lewis, D. (1983). New work for a theory of universals. Australasian Journal of Philosophy, 61, 343-377.

Lewis, D. (1994). Humean supervenience debugged. Mind, 103, 473-490.

Loew, C., \& Jaag, S. (2020). Humean laws and (nested) counterfactuals. The Philosophical Quarterly, 70, 93-113.

Loewer, B. (2007). Laws and natural properties. Philosophical Topics, 35, 313-328.

Loewer, B. (2012). Two accounts of law and time. Philosophical Studies, 160, 115-137.

Marshall, D. (2015). Humean laws and explanation. Philosophical Studies, 172, 3145-3165.

Maudlin, T. (2007). The metaphysics within physics. Oxford: Oxford University Press.

Miller, E. (2015). Humean scientific explanation. Philosophical Studies, 172, 1311-1332.

Nagel, E. (1961). The structure of science: Problems in the logic of explanation. New York: Harcourt, Brace \& World Inc.

Paul, L. A. (2013). Categorical priority and categorical collapse. Proceedings of the Aristotelian Society, Supplementary Volumes, 87, 89-113.

Reutlinger, A. (2018). Extending the counterfactual theory of explanation. In A. Reutlinger \& J. Saatsi (Eds.), Explanation beyond causation. Oxford: Oxford University Press.

Roberts, J. (2008). The law-governed universe. New York: Oxford University Press.

Rosen, G. (2010). Metaphysical dependence: Grounding and reduction. In B. Hale \& A. Hoffmann (Eds.), Modality: Metaphysics, logic and epistemology (pp. 109-136). Oxford: Oxford University Press.

Roski, S. (2018). Grounding and the explanatory role of generalizations. Philosophical Studies, 175, $1985-2003$.

Saatsi, J., \& Pexton, M. (2013). Reassessing Woodward's account of explanation: Regularities, counterfactuals, and non-causal explanations. Philosophy of Science, 80, 613-624. 
Woodward, J. (2003). Making things happen: A theory of causal explanation. New York: Oxford University Press.

Woodward, J. (2017). Scientific explanation. In E. N. Zalta (Ed.), The Stanford encyclopedia of philosophy. https://plato.stanford.edu/archives/fall2017/entries/scientific-explanation/.

Publisher's Note Springer Nature remains neutral with regard to jurisdictional claims in published maps and institutional affiliations. 